\title{
Fadiga: uma análise do conceito
}

\author{
Fatigue: a concept analyses \\ Fatiga: un análisis del concepto \\ Dálete Delalibera Corrêa de Faria Mota', Diná de Almeida Lopes Monteiro da Cruz², \\ Cibele Andrucioli de Mattos Pimenta ${ }^{3}$
}

\begin{abstract}
RESUMO
Introdução: Fadiga é um dos fenômenos mais comumente vivenciados por todos os seres humanos. No entanto, esse conceito é causa de grande confusão na ciência. Objetivo: Analisar o conceito de fadiga. Método: Foi utilizado o método proposto por Walker e Avant (1995). Resultados: São identificados atributos críticos da fadiga e apontados antecedentes, conseqüentes e referências empíricas. Para ilustrar, são apresentados caso-modelo, caso-contrário e caso-ilegítimo. A manifestação de cansaço/canseira ou exaustão associada ao prejuízo no desenvolvimento das atividades usuais do dia-a dia e a falta de resultados das estratégias habituais de recuperação de energia são identificadas como as principais referências empíricas do conceito de fadiga para a área da saúde.Conclusões: Esta análise conceitual oferece base para o desenvolvimento de uma definição operacional e novos direcionamentos para pesquisas. Descritores: fadiga, formação de conceito, pesquisa
\end{abstract}

Descritores: Fadiga; Formação de conceito; Pesquisa

\section{ABSTRACT}

Background: Fatigue is one of the most commonly experienced phenomena by all human being. However, this concept is cause of great confusion in science. Objective: This study analyzed the concept of fatigue using the method of Walker and Avant (1995). Critical attributes, antecedents, consequents and empirical referents are identified. To illustrate the concept, model, contrary and illegitimate cases are presented. Results: The manifestation of tiredness or exhaustion associated with the impact on the development of every day activities and the absence of restorage of energy using habitual strategies are identified as the main empirical referents related to the concept of fatigue. Conclusions: This conceptual analysis offers support for the development of an operational definition and suggests new guidelines for future research.

Keywords: Fatigue; Concept formation; Research

\section{RESUMEN}

Introdución: El fenómeno fatiga es una experiencia común a todos los seres humanos. No obstante, el concepto genera gran confusión en la ciencia. Objetivo: Este estudio analiza el concepto fatiga a través del método propuesto por Walker y Avant (1995). Resultados: Se identifican atributos críticos de fatiga y se destacan los antecedentes, las consecuencias y las referencias empíricas. Para ilustrar, se presenta un caso-modelo, un caso-contrario y un caso-ilegítimo. La manifestación de cansancio o desaliento que perjudica el desarrollo de actividades cotidianas y la falta de resultados ante las estrategias habituales para recuperar el aliento, son identificadas como las principales referencias empíricas del concepto de fatiga en el área de la salud. Conclusiónes: Este análisis conceptual ofrece bases para desarrollar una nueva definición operacional y propone directrices para realizar nuevas investigaciones.

Descriptores: Fatiga; Formación de conceptos; Investigación

\section{INTRODUÇÃO}

Fadiga é um conceito que tem incomodado os profissionais da área da saúde. Tem sido incluída como variável em diversos estudos, provavelmente pela sua alta prevalência nas mais diversas populações e pelo prejuízo que causa à qualidade de vida. Contudo, pode-se observar que nem sempre os autores expõem a concepção que norteou a operacionalização da fadiga como variável em suas investigações. Quando essa exposição ocorre, ou quando é possível apreendê-la, observa-se que não há concordância entre os autores sobre a concepção de fadiga.

Essas observações motivaram a realização deste estudo que analisa o conceito de fadiga. Justificamos a importância dos conceitos para a pesquisa e apresenta-

\footnotetext{
1 Mestre em Enfermagem e Doutoranda do Programa de Pós-Graduação em Enfermagem na Saúde do Adulto da Escola de Enfermagem, Universidade de São Paulo - USP - São Paulo (SP), Brasil.

2 Professora Livre-Docente do Departamento de Enfermagem Médico-Cirúrgica da Escola de Enfermagem, Universidade de São Paulo - USP - São Paulo (SP), Brasil..

3 Professora Titular do Departamento de Enfermagem Médico-Cirúrgica da Escola de Enfermagem, Universidade de São Paulo - USP - São Paulo (SP), Brasil..
} 
mos uma análise do conceito de fadiga segundo o método de Walker e Avant ${ }^{(1)}$.

\section{Importância dos conceitos para a pesquisa}

Há extensas discussões e diferentes perspectivas sobre o que vem a ser um conceito $^{(2)}$. Alguns autores afirmam que os conceitos são, essencialmente, símbolos para elementos objetivos do mundo ${ }^{(1)}$. Outros autores discutem o assunto focalizando-o na mente, no pensamento humano. Afirmam, por exemplo, que conceito é uma imagem mental, uma palavra que simboliza idéias e significados e expressa uma abstração ${ }^{(3)}$. Ainda, há uma terceira perspectiva em que o conceito é discutido especificamente em referência à linguagem. Nessa perspectiva um conceito é simplesmente uma palavra à qual se vincula um significado através de definição formal ou através do uso comum ${ }^{(4-5)}$.

Simplificando as possíveis discussões sobre o que vem a ser um conceito, assumimos para este trabalho que conceito é a imagem mental de um fenômeno; é a idéia que temos na mente sobre uma coisa ou sobre uma ação ${ }^{(1)}$. Conceito é um pensamento, uma noção ou uma idéia $^{(6)}$. Usamos a linguagem para expressar conceitos e assim atribuímos nomes, títulos ou palavras aos conceitos para comunicar nossas idéias. As palavras que usamos não são os conceitos, são apenas instrumentos para comunicá-los ${ }^{(1)}$. Como somos dependentes da linguagem para comunicar nossas idéias, e mesmo para pensar, muitas vezes é difícil distinguir a idéia de um objeto - o conceito da palavra que usamos para expressá-lo.

Um dos principais requisitos da palavra que designa o conceito é que ela seja capaz de expressar através de seu significado o que ocorre na realidade empírica ${ }^{(5)}$, isto é, a(s) palavra(s) usada(s) para designar um conceito deve(m) significar idéias muito semelhantes para diferentes pessoas; deve $(\mathrm{m})$ refletir o mesmo conceito para diferentes pessoas.

Os conceitos são particularmente importantes para a construção do conhecimento. É muito comum para quem já estudou teorias e estruturas conceituais na enfermagem a idéia de que os conceitos são os "tijolos" para construir teorias. A investigação científica trata de explorar ou testar possíveis articulações entre esses "tijolos" com a finalidade de produzir evidências que confirmem, neguem ou modifiquem as teorias.

Toda a atividade de investigação científica lida com conceitos. Ao se desenvolver uma pesquisa qualitativa o objeto em estudo pode ser colocado na perspectiva de conceito; na pesquisa quantitativa, as variáveis sob estudo são também conceitos. A questão dos conceitos é essencial nas pesquisas quantitativas independentemente do grau de abstração das variáveis envolvidas. Se o pesquisador não operacionalizar cuidadosamente o(s) conceito(s) de interesse, dificilmente os resultados de sua pesquisa poderão contribuir para o conhecimento em construção, mesmo que o delineamento utilizado seja adequado.

Operacionalizar um conceito é traduzi-lo em eventos ou fenômenos observáveis ${ }^{(6)}$. Para aplicar conceitos nas investigações quantitativas é necessário que sejam desenvolvidos instrumentos de medidas coerentes com os eventos ou fenômenos observáveis obtidos da sua operacionalização. Muitos dos conceitos pertinentes à saúde têm alto grau de abstração, são complexos, têm várias dimensões e, para complicar, não são estáticos, estão continuamente incorporando novos conhecimentos, experiências, percepções e dados ${ }^{(7)}$. Por isso, o processo de operacionalizar conceitos para a pesquisa, incluindo o desenvolvimento de instrumentos para mensurá-los, consome considerável quantia de recursos e exige que o pesquisador se debruce sobre as questões teóricas e conceituais envolvidas. O estágio final da operacionalização de um conceito para a pesquisa é a construção de um instrumento válido para mensurá-lo.

Uma infinidade de instrumentos de medidas de variáveis psicossociais tem sido produzida. Freqüentemente, para um mesmo fenômeno há diversos instrumentos de medida desenvolvidos. O pesquisador, em busca da melhor instrumentação para o seu estudo, tem que decidir entre os instrumentos existentes, ou ainda, se deveria desenvolver um novo instrumento.

Várias características dos instrumentos disponíveis devem ser analisadas para guiar a seleção do mais adequado para o interesse do pesquisador. $\mathrm{O}$ pesquisador deve estar atento às perspectivas teóricas que os autores dos instrumentos utilizaram para desenvolvê-los. Nem sempre os autores expõem claramente sequer uma definição teórica do conceito avaliado pelo instrumento, podendo ser um obstáculo para os pesquisadores usarem esse instrumento.

A análise de conceitos pode ser estratégia para descobrir o delineamento do fenômeno. Os resultados desse processo são úteis para julgar os instrumentos de medida disponíveis quanto à capacidade de cobrir o fenômeno em questão, a construção de instrumentos de medida, e também para permitir a observação e crítica de outros interessados no mesmo conceito.

\section{$O$ conceito de fadiga}

Exploração inicial sobre fadiga permitiu observar falta de consistência entre os autores sobre o conceito e, também, que poucos autores expressam preocupação com a perspectiva teórica sob a qual o fenômeno é observado. Buscando facilitar a promoção do conhecimento relacionado ao fenômeno, este estudo teve como objetivo analisar o conceito de fadiga para determinar seus atri- 
butos críticos, antecedentes e conseqüentes a fim de estabelecer fundamentos para a aplicação do conceito em pesquisas.

Poderíamos dizer que fadiga ainda é um conceito imaturo, ou seja, um conceito sobre o qual há quantida-

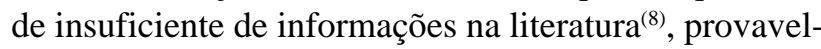
mente devido a pouca quantidade de pesquisas dedicadas ao entendimento do fenômeno. A falta de compreensão comum sobre fadiga retarda a construção de novo conhecimento a ela relacionado. Certa dose de consenso sobre o significado dos conceitos é importante para o estabelecimento de hipóteses testáveis. Submeter essas hipóteses a testes permite avançar o conhecimento.

Fadiga é um fenômeno relatado desde a antiguidade. O termo é usado na Bíblia relacionando-o a sofrimento por causas físicas (trabalho excessivo) ou mentais (sentimento de culpa) ${ }^{(9)}$. É um termo derivado do latim 'fatigãre' e o uso na língua portuguesa data de $1844^{(10)}$. Além de ser antiga, a idéia de fadiga está presente em várias situações, envolvendo tanto objetos como seres humanos. Nos seres humanos, o conceito fadiga precisa ser refinado e melhor compreendido pelos profissionais de saúde. Como a fadiga é um fenômeno que interessa a várias disciplinas da área da saúde, há dificuldade na obtenção do consenso, uma vez que cada profissional tende a definir fadiga segundo a sua perspectiva. Por exemplo, para pesquisadores que estudam fadiga relacionada à atividade física, associa-se o fenômeno mais às causas físicas. Por outro lado, aqueles que estudam fadiga relacionada a distúrbios psiquiátricos, associamna mais às causas mentais. Mesmo não havendo um consenso quanto ao conceito de fadiga, grande parte dos pesquisadores concorda que é um fenômeno subjetivo, multicausal, cuja gênese e expressão envolvem aspectos físicos, cognitivos e emocionais ${ }^{(11)}$.

No início do século XX admitiu-se que a fadiga não podia ser estudada por causa de sua complexidade conceitual e só a partir da década de 1950 que se tornou novamente tópico de interesse ${ }^{(12)}$. Atualmente, frente à magnitude da fadiga no âmbito da saúde, a análise do conceito de fadiga assume grande importância. Como a fadiga é comum e importante para diversos cenários de atenção à saúde, são importantes também os estudos que produzem conhecimentos úteis ao diagnóstico, mensuração e tratamento do fenômeno.

\section{Análise de conceito}

A ênfase no desenvolvimento de conceito cresceu muito na década de 1990 tanto para a enfermagem como para outras áreas. Hoje é visto como uma forma de investigação significativa para o avanço do conhecimento em várias disciplinas ${ }^{(2)}$.
Os conceitos são definidos e analisados de numerosas formas por diferentes autores ${ }^{(6)} \mathrm{e}$, devido a alguma confusão, o desenvolvimento de conceito tem sido aplicado como sinônimo de análise e de clarificação de conceito $^{(7)}$. Os conceitos podem ser desenvolvidos aplicando-se diferentes estratégias e as principais são: exploração de conceito, análise de conceito e clarificação de conceito $^{(7)}$. Exploração de conceito é estratégia usada quando novos conceitos são identificados antes de eles serem aceitos como componentes da terminologia da enfermagem ${ }^{(7)}$. A clarificação de conceito pode ser usada para refinar conceitos que são aplicados na enfermagem sem um acordo claro, compartilhado e consciente sobre as propriedades ou significados a eles atribuídos ${ }^{(7)}$. Ao usar a análise de conceito, o pressuposto é que o conceito foi introduzido na literatura, foi definido e clarificado, mas que há necessidade de mais estudos para que se alcance um outro estágio de desenvolvimento. Realiza-se análise de conceito quando sua significância para enfermagem está estabelecida e sua relação com a disciplina foi esclarecida ${ }^{(7)}$.

O conceito de fadiga foi aceito pela North American Nursing Diagnosis Association como um diagnóstico em 1988 e revisado em $1998^{(13)}$. Com base nesse dado, associado à observação de que o conceito é freqüentemente aplicado na clínica de enfermagem, admitimos que a análise de conceito é uma estratégia adequada ao conceito de fadiga.

A análise de conceito tem a finalidade de delinear atributos ou características do fenômeno estudado e, além disso, manter o conceito atualizado, visto que os conceitos vão continuamente se modificando ${ }^{(2,7)}$. Fazer análise de conceito implica em examiná-lo profundamente e, implica também em diferenciar os atributos definidores do conceito de outros atributos irrelevantes. Nesse caso, isso significa detectar diferenças entre fadiga e outros conceitos semelhantes, como, por exemplo, o de, astenia.

Inúmeras estratégias de análise de conceito estão presentes na literatura ${ }^{(1-2,7,14-16)}$. Dentre esses, a maioria surgiu como adaptação ou crítica à técnica proposta por Wilson em $1963^{(7)}$. Apesar de algumas técnicas, como a proposta nos estudos ${ }^{(2,16)}$ serem mais atuais e consideradas mais modernas, aprimoradas e avançadas, optamos por utilizar a técnica proposta por Walker e Avant ${ }^{(1)}$, primeiro descrita em 1983. Escolhemos esse método porque, além de ser a técnica de análise mais tradicional, é uma técnica clara, simples e de fácil aplicação.

\section{MÉTODO}

A técnica de Walker e Avant ${ }^{(1)}$ compreende 8 etapas adaptadas das 11 sugeridas por Wilson em $1963^{(17)}$. Apesar de alguns passos ocorrerem simultaneamente, eles serão descritos seqüencialmente para facilitar a compre- 
ensão pelos leitores. Os resultados das duas primeiras etapas estão incorporados na descrição do método porque eram necessários para a definição das etapas subseqüentes. As descrições das etapas foram extraídas da proposta de Walker e Avant ${ }^{(1)}$.

\section{Seleção do conceito}

Esta primeira etapa seria uma das mais difíceis visto que a escolha de um conceito para análise implica num exercício formal, rígido e que demanda tempo e dedicação ${ }^{(1)}$. Porém, a seleção do conceito fadiga para nós foi uma das etapas mais simples visto que fadiga já era tema de estudo e esse exercício seria útil e importante para pesquisas futuras.

\section{Objetivos da análise}

A análise do conceito pode ter diferentes objetivos: esclarecer o significado de um conceito, desenvolver uma definição operacional e adicionar/renovar uma teoria existente, entre outros. Neste estudo, a análise do conceito de fadiga teve como propósito único o esclarecimento desse fenômeno na área da saúde.

\section{Identificação dos usos do conceito}

Nessa etapa, os autores sugerem identificar a maior variedade possível de aplicações do conceito e considerálas todas ${ }^{(1)}$. Referem, ainda, que a busca do uso do conceito apenas em literatura médica ou o fato de ignorar a existência de outros usos do conceito podem causar vieses na compreensão da sua natureza e limitação na utilidade dos resultados da análise.

Para identificar os diversos usos do conceito fadiga foram utilizados: artigos de periódicos, livros textos e dicionários. Os artigos foram obtidos através de consulta às bases de dados do MEDLINE, LILACS e no banco de dados DEDALUS, usando a palavra-chave fadiga (fatigue). Os livros textos e dicionários foram obtidos através de busca nos acervos da biblioteca da BIREME e biblioteca da Escola de Enfermagem da Universidade de São Paulo.

\section{Determinação dos atributos definidores}

Os atributos definidores, também chamados de atributos críticos, são características que atuam como elementos para diagnósticos diferenciais, isto é para discriminar o que é uma expressão do conceito do que não é(1). Os atributos podem variar de acordo com o contexto em que estão inseridos e, portanto, devemos identificar os atributos que estão mais freqüentemente associados com o conceito, nos mais distintos contextos possíveis ${ }^{(1)}$.

\section{Desenvolvimento de casos-modelo}

Essa etapa visa ilustrar o conceito através de um exemplo que contenha os atributos definidores do mesmo. O caso deve ser o mais paradigmático possível ${ }^{(1)}$.

\section{Desenvolvimento de outros casos}

Casos-limítrofes, casos-relacionados, casos-contrários, casos-inventados, casos ilegítimos são exemplos de casos adicionais. Esses são relatados durante a análise de conceito para auxiliar na decisão quanto aos atributos realmente importantes para o conceito. Todos esses casos não são exemplos legítimos do conceito.

\section{Identificação de antecedentes e conseqüentes}

A identificação de antecedentes e conseqüentes do conceito, como o próprio nome diz, é o levantamento de incidentes ou eventos que acontecem a priori e posteriori ao fenômeno, respectivamente. Eles podem coincidir ou não com os atributos definidores.

\section{Definição de referências empíricas}

Esse é o passo final da análise de conceito, na qual se busca identificar referências empíricas para os atributos definidores. Em muitos casos, os atributos definidores ou atributos críticos serão idênticos às referências empíricas. Nos casos em que o conceito é muito abstrato, os seus atributos definidores geralmente têm também alto grau de abstração. Nessas situações é necessário que os atributos definidores sejam decodificados em referentes empíricos. Os referentes empíricos são categorias ou classes de fenômenos observáveis que, quando presentes, demonstram a ocorrência do conceito. No caso da fadiga, as referências empíricas seriam as manifestações observáveis na pessoa com fadiga.

\section{APRESENTAÇÃO E DISCUSSÃO DOS RESULTADOS}

\section{Usos do conceito}

Com o levantamento bibliográfico identificamos que fadiga é um conceito presente numa ampla gama de disciplinas acadêmicas. Nas áreas relacionadas à saúde, fadiga está presente na medicina, educação física, psicologia, enfermagem, farmacologia, nutrição, odontologia, ergonomia e genética. $\mathrm{O}$ conceito de fadiga em áreas não relacionadas à saúde, como engenharia, foi levantado pelo banco de dados DEDALUS. Com a finalidade de abranger o maior número de contextos possíveis nos quais o conceito fadiga está presente, todas as definições foram incluídas na análise e as principais definições estão apresentadas no Quadro 1. 
Quadro 1 - Apresentação de diversas definições do conceito fadiga presentes na literatura

\begin{tabular}{|c|c|}
\hline Área & Definição de fadiga \\
\hline Psicologia ${ }^{(36)}$ & "estado de desgaste relacionado a redução da motivação" \\
\hline Enfermagem $(13,38,39)$ & $\begin{array}{l}\text { "uma sensação opressiva e sustentada de exaustão e de capacidade diminuída para realizar trabalho } \\
\text { físico e mental no nível habitual" } \\
\text { "sintoma subjetivo, desagradável que incorpora toda sensação do corpo variando de cansaço a exaustão, } \\
\text { criando uma condição geral de falta de alívio, que interfere na capacidade do indivíduo realizar suas } \\
\text { habilidades normais" } \\
\text { "experiência aguda ou crônica caracterizada pelo desempenho não efetivo de tarefas, inadequação } \\
\text { auto-percebida, aversão à atividades, cansaço ou sensação de fraqueza, desconforto" }\end{array}$ \\
\hline Educação Física ${ }^{(37)}$ & "declínio na capacidade de gerar tensão muscular com a estimulação repetida" \\
\hline Index Medicus (26) & $\begin{array}{l}\text { "estado de desgaste que segue um período de esforço, mental ou físico, caracterizado por uma } \\
\text { diminuição da capacidade de trabalhar e redução da eficiência para responder a um estímulo" }\end{array}$ \\
\hline Oncologia ${ }^{(40)}$ & $\begin{array}{l}\text { "condição caracterizada por sofrimento e diminuição da capacidade funcional devido a redução de } \\
\text { energia" }\end{array}$ \\
\hline Odontologia ${ }^{(41)}$ & $\begin{array}{l}\text { A fadiga refere-se ao 'desgaste' de materiais de próteses parciais, geralmente gerado por falha no próprio } \\
\text { material ou por efeito de cargas repetidas }\end{array}$ \\
\hline Engenharia ${ }^{(42)}$ & Fadiga é um modo prevalente de falência de componentes estruturais ocasionado por períodos de estresse \\
\hline Dicionário etimológico ${ }^{(10)}$ & 'cansaço' \\
\hline $\begin{array}{l}\text { Dorland's illustrated } \\
\text { Medical Dictionary }{ }^{(21)}\end{array}$ & $\begin{array}{l}\text { 'estado com aumento do desconforto e diminuição da eficiência resultante de um esforço prolongado } \\
\text { ou excessivo' }\end{array}$ \\
\hline
\end{tabular}

Embora difícil, é possível traçar algumas semelhanças entre as definições assumidas na atualidade. Todavia, alguns autores consideram a definição de fadiga uma ilusão devido a multidimensionalidade do fenômeno ${ }^{(18)}$. Para outros, a falta de atenção na diferenciação entre as características da fadiga e as de outros conceitos é uma das causas históricas para a falta de consenso na definição da fadiga ${ }^{(19)}$. Consideramos que o maior problema seja a tentativa de incluir causas ou fatores relacionados nas definições de fadiga, buscando atender a todas as situações nas quais o fenômeno pode estar presente. No entanto, isso causa grande confusão visto que o fator causal do fenômeno pode ser diferente em cada situação de fadiga.

\section{Atributos definidores}

Após a identificação dos usos do conceito e análise das definições, destacaram-se os seguintes atributos: cansaço, exaustão, desgaste, fraqueza, astenia, diminuição da capacidade funcional ou da capacidade de realizar atividades diárias,falta de recursos/energia/capacidade latente, redução da eficiência para responder a um estímulo, desconforto, sonolência, diminuição da motivação, aversão a atividades, sofrimento e necessidade extrema de descanso.
Buscou-se a definição de cada atributo para aprofundar a análise dos mesmos. A primeira busca foi feita em dicionários médicos ${ }^{(20-22)}$, porém não foram encontradas definições para a maioria dos atributos. Encontraram-se apenas as que, na verdade, se referem a sinais ou sintomas reconhecidos pela medicina/enfermagem como diagnosticáveis. Nesses dicionários, os atributos encontrados foram: exaustão, astenia, letargia e sonolência. Portanto, em seguida, partiu-se para a busca em outros dicionários ${ }^{(10,23-24)}$. Nesses, os atributos encontrados foram fraqueza, cansaço, energia, motivação, atenção, desconforto, esforço, desgaste, além dos atributos também encontrados nos dicionários médicos (astenia, exaustão, letargia e sonolência).

As definições dos atributos cansaço, exaustão, desgaste, alteração da capacidade funcional e falta de recursos/energia mostram-se como os principais atributos da fadiga, independente da disciplina que estuda o fenômeno. Isso é muito claro devido a forte relação entre essas definições e a maioria das definições propostas para o conceito fadiga.

Outros atributos como letargia, sonolência, diminuição da motivação, atenção e concentração, necessidade extrema de descanso e mal-estar, seriam mais bem classificados como antecedentes ou conseqüentes de fadiga. Isso se deve ao fato de esses fenômenos não serem 
essenciais para que fadiga seja atribuída a um indivíduo, visto que nem sempre estão presentes num mesmo momento ou com a mesma intensidade ${ }^{(25)}$.

No entanto, outros atributos como fraqueza, falta de energia/força, desconforto, esforço, aversão a atividades seriam melhor aplicados ao conceito de astenia por serem definidos de forma mais coerente com a definição desse conceito. Nos dicionários médicos astenia é definida como fraqueza, debilidade, um sinal ou sintoma clínico manifestado por incapacidade ou falta/perda de força e energia ${ }^{(26)}$, enquanto que fadiga é definida como

estado de desgaste que segue um período de esforço, mental ou físico, caracterizado por diminuição da capacidade de trabalhar e redução da eficiência para responder a um estímulo ${ }^{(26)}$.

Durante a análise da literatura, notou-se que muitos autores ainda utilizam como sinônimos de fadiga os termos astenia, letargia e sonolência ${ }^{(18,27-28)}$. Nessas situações, os autores sequer apresentaram definições para os conceitos que propõem pesquisar. É preciso deixar claro que os atributos da fadiga estão se modificando em relação ao conhecimento que havia no início da década de 80. Esse movimento é natural porque ocorre uma evolução do conceito devido ao avanço do conhecimento ${ }^{(2,7)}$. Portanto, a diferenciação entre fadiga e astenia é, atualmente, mais nítida como pode ser observado pela comparação entre as definições de fadiga e de astenia apresentadas neste estudo.

\section{Caso-modelo}

Como modelo para fadiga citamos o seguinte caso:

Executivo, com diagnóstico recente de câncer de cabeça e pescoço, após x sessões de radioterapia, começa a apresentar necessidade de deixar o trabalho mais cedo que o habitual e não consegue mais desenvolver todas as atividades domésticas pelas quais era responsável, por sentir-se muito cansado. Mantém-se em repouso, buscando descanso, durante grande parte do dia e refere que mesmo assim não consegue recuperar a disposição e energia necessárias para o funcionamento habitual prévio.

Por ser um fenômeno subjetivo, os atributos só podem ser identificados através de menção pelo sujeito fatigado ou por comportamentos, que seriam as referências empíricas. Assim, esse caso apresenta todos os atributos críticos da fadiga: cansaço ou exaustão, alteração na capacidade funcional desgaste e falta de alívio para essas manifestações com a aplicação de estratégias usuais de recuperação de energia.

\section{Outros casos}

Como exemplo de casos adicionais serão apresentados um caso-contrário e um caso-ilegítimo.

\section{Caso-contrário:}

Mulher de meia-idade, após uma boa noite de sono, acorda no horário habitual e realiza todas as atividades de vida diária que costuma realizar rotineiramente e refere sentir-se disposta e com a energia habitual.

\section{Caso-ilegítimo:}

No período de férias escolares, um adolescente saudável, após dormir mais de 12 horas seguidas, acorda e permanece durante toda a manhã deitado no sofá, assistindo televisão. Sua mãe pede para que arrume o quarto, porém ele permanece deitado, inativo e refere que quer descansar das intensas atividades do período letivo.

No primeiro caso, fica claro que a mulher não apresenta fadiga visto que suas manifestações indicam que a energia que percebe é adequada às demandas que experimenta. Já no segundo, apesar de o adolescente não reagir à solicitação da atividade, não há manifestações de que a demanda por atividade é superior a energia que possui. Além disso, não há antecedentes e consequientes lógicos para fadiga, aparentando ser simplesmente um caso de preguiça ou de preferência por uma ou outra atividade.

\section{Antecedentes e conseqüientes}

Como mencionado anteriormente, muitos dos atributos de fadiga deveriam ser classificados como antecedentes ou conseqüentes do fenômeno. Reconhecendo antecedentes e consequientes da fadiga, facilita-se a determinação de variáveis a serem estudadas no futuro.

Os antecedentes de fadiga podem ser os mais diversos como, por exemplo, atividade física intensa, esforço prolongado ou excessivo, depressão, gestação e parto, presença de doenças e distúrbios do sono. Esses inúmeros antecedentes da fadiga tendem a aumentar com a realização de novos estudos que resultam em descobertas quanto às causas do fenômeno.

Os consequientes seriam letargia, sonolência, diminuição da motivação, atenção, paciência e concentração, sofrimento, necessidade extrema de descanso e mal-estar. Vale mencionar que as implicações dos conseqüentes para o indivíduo fatigado são evidentes e são os principais focos de ação dos profissionais. Os conseqüentes produzem sofrimento e devem direcionar o manejo e controle do fenômeno. 


\section{Referências empíricas}

Como mencionado no método, as referências empíricas são características observáveis que indicam a presença de fadiga sendo, essas características, ilustrações dos atributos críticos e conseqüentes. Muitos instrumentos desenvolvidos para avaliar fadiga são baseados nessas referências pois a fadiga em si não é observável. A partir de diversos instrumentos, as referências mais aceitas no momento são: capacidade de quantificar a fadiga, impacto da fadiga na capacidade de realizar atividades de vida diária, necessidade de descanso, verbalização da sensação de cansaço/canseira/ exaustão, sensação de angústia, atividade física como causa ou fator agravante de fadiga, interferência no relacionamento interpessoal, socialização, atividade sexual e humor, redução da motivação, dificuldade em manter concentração e atenção, e prejuízos de memória ${ }^{(29-33)}$.

Não podemos deixar de observar três aspectos importantes nesses referentes de fadiga. O primeiro é que eles indicam a subjetividade da fadiga. Isto é, a maior parte dos seus referentes só pode ser identificada por auto-relato. $\mathrm{O}$ segundo é a circularidade existente entre alguns referentes e a própria fadiga. Por exemplo, a "capacidade de quantificar a fadiga" é um referente que admite qualquer concepção de fadiga pela pessoa que a quantifica, de forma que pouco contribui para a operacionalização do conceito de fadiga. Essas observações tenderiam a fundamentar a idéia de que fadiga não pode ser operacionalizada. $\mathrm{O}$ terceiro aspecto a ser destacado nos referentes identificados é que "a verbalização de canseira/cansaço ou exaustão" é um referente que não apresenta circularidade com a fadiga e nem é seu antecedente ou consequente. Esse referente nos parece o mais característico e por isso o mais útil para a operacionalização do conceito. Mas, tomando como exemplo o caso ilegítimo apresentado, podemos observar que houve verbalização de cansaço pelo adolescente. O que diferencia "a verbalização de canseira/cansaço ou exaustão" entre o caso-modelo e o caso ilegítimo apresentados é que, no caso-modelo, observa-se prejuízo no desempenho das atividades diárias usais e as estratégias de recuperação de energia não produzem o resultado esperado.

Essa análise nos permite afirmar que três referentes empíricos associados são os mais importantes para o conceito de fadiga: a verbalização de cansaço/canseira ou exaustão, diminuição da capacidade de realizar atividades habituais e a falta de alívio para essas manifestações com a aplicação de estratégias usuais de recuperação de energia. Os outros referentes relativos aos antecendentes e conseqüentes da fadiga são complementares e as pesquisas deverão mostrar a importância deles na operacionalização do conceito, contribuindo para o desenvolvimento de teorias em que a fadiga é conceito constituinte.

Cabe relembrar que, como o conceito fadiga tem sido de difícil definição, sua mensuração também é complicada, principalmente por ser um fenômeno subjetivo. Mas, assim como o conceito está sendo refinado, os instrumentos também o serão acompanhando o conhecimento gerado pela pesquisa.

\section{CONCLUSÃO}

A análise do conceito de fadiga permitiu observar que muitos outros conceitos estão amarrados ao seu. Esse fato muito dificulta a determinação dos atributos críticos da fadiga, pois outros como fraqueza, estão arraigados desde seus primeiros relatos na literatura.

Parece que, por muitas vezes estarem presentes concomitantemente em indivíduos doentes, os conceitos de fadiga, astenia, letargia, sonolência, falta de motivação estão mesclados nas mentes dos profissionais e, com isso, seus respectivos atributos não estão claros. Assim, o diagnóstico de apenas um desses fenômenos torna-se mais difícil, pois não se sabe qual atributo reflete qual conceito. Contudo, se não reconhecermos as especificidades de cada fenômeno, em especial da fadiga, o desenvolvimento de conhecimento sobre o tema ocorrerá de forma extremamente lenta. Sem a compreensão do conceito de fadiga, cada pesquisador estará construindo conhecimento somente para sua realidade, pois os resultados das pesquisas não poderão ser compartilhados e articulados.

Assumindo, nesse momento, a fadiga de modo simples, como foi proposto neste estudo, o conceito tornase aplicável em qualquer espaço no cenário da saúde. A partir disso, é possível traçar novos direcionamentos para o desenvolvimento de estudos buscando aprofundar o conhecimento sobre fadiga em diferentes situações, pois quanto mais claramente um conceito é definido, maior é a sua utilidade como variável de pesquisa. Somando-se a isso, num futuro próximo poderão ser feitas definições mais específicas de fadiga com levantamento de atributos específicos para cada situação na qual a fadiga está presente. Na realidade, de forma discreta, isso já está ocorrendo. É o caso do conceito de fadiga pósparto $^{(34)}$.

A técnica de Walker e Avant ${ }^{(1)}$, utilizada para análise do conceito de fadiga neste estudo, embora simples e de fácil aplicação, mostrou-se mais clara após o estudo da técnica original proposta por Wilson em $1963^{(17)}$. Apesar de o número de passos ser menor na proposta de Walker e Avant, todo o processo possui muitas semelhanças.

O presente estudo alcançou resultados semelhantes aos de Trendall ${ }^{(35)}$, que usou a mesma metodologia para analisar o conceito de fadiga crônica. Resultados da apli- 
cação de outras técnicas de análise de conceito, tais como as de Rodgers ${ }^{(2)}$ ou Morse et al. ${ }^{(16)}$ certamente trarão importantes contribuições para refinar o conceito de fadiga.

\section{REFERÊNCIAS}

1. Walker LO, Avant KC. Concept development. In: Walker LO, Avant KC. Strategies for theory construction in nursing. 3rd ed. Norwalk: Appleton \& Lange; 1995. p. 35-78.

2. Rodgers BL. Philosophical foundations of concept development. In: Rodgers BL, Knafl KA. Concept development in nursing foundations, techniques, and applications. 2nd ed. Philadelphia: Saunders; 2000, p. 7-37.

3. Watson J. Nursing: the philosophy and science of caring. Boston: Little Brown; 1979.

4. Diers D. Research in nursing practice. Philadelphia: Lippincott; 1979.

5. Mendonça ND. O uso dos conceitos: uma questão de interdisciplinaridade. 4a ed. Petrópolis: Vozes; 1985.

6. Waltz CF, Strickland OL, Lenz ER. Measurement in nursing research. 2nd ed. Philadelphia: Davis; 1991.

7. Meleis AI. Strategies for concept development. In: Meleis AI. Theoretical nursing: development and progress. Philadelphia: Lippincott; 1997. p. 203-22.

8. Gift AG, editor. Clarifying concepts in nursing research. New York: Springer; 1997.

9. Bíblia Sagrada. Traduzida por Almeida JF. Brasília: Sociedade Bíblica do Brasil; 1990.

10. Cunha AG. Dicionário etimológico da língua portuguesa. 2a ed. Rio de Janeiro: Nova Fronteira; 1999.

11. Mota DDCF, Pimenta CAM. Fadiga em pacientes com câncer avançado: conceito, avaliação e intervenção. Rev Bras Cancerol. 2002; 48(4):577-83.

12. Winningham ML. Fatigue. In: Groenwald SL, Frogge MH, Goodman M, Yarbro CH. Cancer symptom management. Boston: Jones and Bartlett; 1996. p. 42-53.

13. North American Nursing Diagnosis Association (NANDA). Nursing diagnoses: definitions and classification 2003-2004. Philadelphia: NANDA Internacional; 2003.p. 74.

14. Wills EM, McEwen M. Concept development: clarifying meaning of terms. In: McEwen M, Wills EM. Theoretical basis for nursing. Philadelphia: Lippincott; 2002. p. 48-68.

15. Swartz-Barcott D, Kim HS. An expansion and elaboration of the hybrid model of concept development. In: Rodgers BL, Knafl KA. Concept development in nursing - foundations, techniques, and applications. 2nd ed. Philadelphia: Saunders; 2000. p. 129-60.
16. Morse JM, Hupcey JE, Mitcham C, Lenz ER. Choosing a strategy for concept analysis in nursing research. In: Gift AG. Clarifying concepts in nursing research. New York: Springer; 1997. p. 73-96.

17. Wilson J. Pensar com conceitos. Traduzido por Barcellos W. São Paulo: Martins Fontes; 2001.

18. Lichstein KL, Means MK, Noe SL, Aguillard RN. Fatigue and sleep disorders. Behav Res Ther. 1997;35(8):733-40.

19. Aaronson LS, Teel CS, Cassmeyer V, Neuberger GB, Pallikkathayil L, Pierce J, et al. Defining and measuring fatigue. Image J Nurs Schol. 1999;31(1):45-50.

20. Anderson KN, Anderson LE, editor. Mosby: dicionário de enfermagem. 2a ed. São Paulo: Roca; 2001.

21. Dorland WAN. The American illustrated medical dictionary. 20th ed. Philadelphia: Saunders; 1981.

22. Stedman TL. Stedman's Medical Dictionary. 25a ed. Baltimore: William and Wilkins; 1990.

23. Pietzschke F, Wimmer F. Novo Michaelis: dicionário ilustrado. 38a ed. São Paulo: Melhoramentos; 1985.

24. Morris W, editor. The American Heritage Dictionary of the English Language. Boston: Houghton Mifflin; 1978.

25. Krishnasamy M. Fatigue in advanced cancer - meaning before measurement? Int J Nurs Stud. 2000;37:401-14.

26. Index Medicus. Washington: NIH Publications; 2002.

27. Sobrero A, Puglisi F, Guglielmi A, Belvedere O, Aprile G, Ramello M, Grossi F. Fatigue: a main component of anemia symptomatology. Semin Oncol. 2001;28(2 Suppl 8):15-8.

28. Tyler LS, Lipman AG. Fatigue in palliative care patients. In: Lipman AG, Jackson KC, Tyler LS. Evidence based symptom control in palliative care: systematic review and validated clinical practive guidelines for 15 common problems in patients with life limiting disease. New York: The Haworth Press; 2000. p.129-140.

29. Mendoza TR, Wang XS, Cleeland CS, Morrissey M, Johnson BA, Wendt JK, Huber SL. The rapid assessment of fatigue severity in cancer patients: use of the brief fatigue inventory. Cancer. 1999;85(5):1187-96.

30. Piper BF, Dibble SL, Dodd MJ, Weiss MC, Slaughter RE, Paul $\mathrm{SM}$. The revised piper fatigue scale: psychometric evaluation in women with breast cancer. Oncol Nurs Forum. 1998; 25(4):677-84.

31. Cella D. The functional Assessment of Cancer Therapy - Anemia (FACT-An) Scale: A new tool for the assessment of outcomes in cancer anemia and fatigue. Semin Hematol. 1997;34(3 Suppl 2):13-9. 
32. Chalder T, Berelowitz G, Pawlikowska T, Watts L, Wessely S, Wright D et al. Development of a fatigue scale. J Psychosom Res. 1993;37(2):147-53.

33. Krupp LB, LaRocca NG, Muir-Nash J, Steinberg AD. The fatigue severity scale: application to patients with multiple sclerosis and systemic lupus erythmatosus. Arch Neurol.1989; 46:1121-3.

34. Milligan R, Lenz ER, Parks PL, Pugh LC, Kitzman H. Pospartum fatigue: clarifying a concept. In: Gift A, editor. Clarifying concepts in nursing research. New York: Springer; 1997.

35. Trendall J. Concept analysis: chronic fatigue. J Adv Nurs. 2000;32 (5):1126-31.

36. Lee KA, Hicks G, Nino-Murcia G. Validity and Reliability of a scale do assess fatigue. Psychiatr Res. 1991;36:299-305

37. McArdle WD, Katch FI, Katch VL. Fisiologia do exercício: energia, nutrição e desempenho humano. Rio de Janeiro: 4a ed. Rio de Janeiro: Guanabara Koogan; 1998. cap. 19. p. 333.
38. Ream E, Richardson A. Fatigue: a concept analysis. Int J Nurs Stud. 1996;33(5):519-29.

39. Varrichio CG. Selecting a tool for measuring fatigue. Oncol Nurs Forum. 1985;12:122-7.

40. National Cancer Institute. Fatigue. [text on the internet]. 2002 [cited 2002 Jun 14]. Available from: www.cancer.gov

41. Meloncini MA, Cardoso PEC, Grande RHM, Muench A. Falhas de grampos circunferenciais de próteses removíveis em função de ciclagens de flexão. Rev Odontol Univ São Paulo. 1998; 12 (3):257-60.

42. Petrucci G, Zuccarello B. Fatigue life prediction under wide band random loading. Fatigue \& Fracture of Engineering Materials \& Structures. 2004;27(12):1183-95. 